Suzana Patrícia de Sá Silva ${ }^{1}$

Gilza Sandre-Pereira ${ }^{2}$

Rosana Salles-Costa ${ }^{2}$

\title{
Fatores sociodemográficos e atividade física de lazer entre homens e mulheres de Duque de Caxias/RJ
}

\author{
Socio-demographic factors and leisure-time physical activity \\ among men and women of Duque de Caxias / RJ
}

${ }^{1}$ Programa de Pós-

Graduação em Nutrição,

Instituto de Nutrição Josué de Castro, Universidade

Federal do Rio de Janeiro.

Av. Carlos Chagas Filho 373

Edifício do Centro de

Ciências da Saúde, Bloco J,

$2^{\circ}$ andar. 21.941-902 Rio de

Janeiro RJ.

supat_sa@hotmail.com

${ }^{2}$ Departamento de Nutrição

Social e Aplicada, Instituto

de Nutrição Josué de Castro,

Universidade Federal do

Rio de Janeiro
Abstract Objectives: To evaluate the prevalence and associated variables in leisure-time physical activity (LTPA), by gender, in Duque de Caxias/ Rio de Janeiro, Brazil. Methods: This is a population-based cross-sectional study with 1,246 adults. During home visits, questionnaires were applied on LTPA, socio-demographic factors and anthropometric examinations were performed. Results: Physical inactivity was high (70\%) (p-value $<0001)$ and men were more active $(43.3 \%)$ than women $(20 \%)$ (p-value $<0.0001)$. Men practiced significantly more competitive and male-associated LTPA. Taking care of children was a factor that increased the likelihood of men engaging in LTPA $(O R=2.75$, $p$-value $=0.034)$, whereas among women, those who spent less time on domestic activities $(O R=0.99, p$-value $=0.016)$, did not smoke $(O R=2.63$, $p$-value $=0.039)$ and had greater accumulation of abdominal fat $(\mathrm{OR}=2.72, p$-value $=0.023)$, practiced LTPA. Conclusion: Men chose LTPAs considered masculine. Socio-demographic and anthropometric measures had different associations with LTPA between the genders.

Key words Leisure-time physical activity, Gender, Household physical activities, Abdominal obesity, Population studies
Resumo Objetivos: Avaliar prevalência, atividades praticadas no tempo destinado ao lazer (AFL) $e$ suas variáveis associadas, entre gêneros, em Duque de Caxias/Rio de Janeiro, Brasil. Material e métodos: Trata-se de um estudo transversal, de base populacional, composto por 1246 adultos. Durante as visitas domiciliares foram aplicados questionários avaliando a prática de AFL, fatores sociodemográficos e medidas antropométricas. Resultados: A inatividade física foi elevada (70\%) ( $p$-valor <0,001) sendo os homens mais ativos (43,3\%) que as mulheres $(20 \%)$ ( $p$-valor < 0,0001). Homens praticaram significativamente mais AFL competitivas e do universo masculino. Tomar conta de crianças foi um fator que aumentou a chance dos homens se engajarem em AFL $(O R=2,75$, $p$-valor $=0,034)$, enquanto que entre as mulheres, aquelas que dispendiam menos tempo em atividades domésticas $(O R=0,99, \quad p$-valor $=0,016)$, não fumavam $(O R=2,63$, p-valor $=0,039)$ e que apresentavam maior acúmulo de gordura abdominal ( $O R=2,72$, p-valor=0,023), tinham mais chance de praticarem AFL. Conclusão: Conclui-se que homens repetiram modelo de escolhas de AFL consideradas masculinas. Fatores sócio-demográficos e medidas antropométricas se associaram com AFL de forma diferente entre gêneros.

Palavras-chaves Atividade física de lazer, Gênero, Atividade física doméstica, Obesidade abdominal, Estudos populacionais 


\section{Introdução}

As alterações no estilo de vida, observadas nos últimos anos, desencadearam um aumento da inatividade física ${ }^{1}$, tornando-se um problema sério em vários lugares do mundo, tanto em países em desenvolvimento quanto nos desenvolvidos ${ }^{2}$, mais comum em classes sociais com pessoas menos instruídas, vivendo abaixo da linha de pobreza e em famílias com menor rendimento ${ }^{3}$.

Estudos internacionais ${ }^{4-6}$ e nacionais ${ }^{7-9}$ revelam que a inatividade física é mais prevalente entre as mulheres quando comparado com os homens e aquelas que pertencem a grupos de menor status socioeconômico teriam maior probabilidade de não praticarem atividades físicas, devido a limitações à prática de exercícios em espaços públicos ${ }^{10}$.

A fim de se entender porque as mulheres são mais inativas em relação aos homens, o conceito de gênero pode ser útil. Este termo, pertencente ao campo das ciências sociais, tem por objetivo estudar as construções sociais sobre os papéis atribuídos pelas sociedades aos homens e às mulheres, como eles se relacionam e suas relações de poder ${ }^{11}$.

E, quando avaliada por meio da dimensão de gênero, a prática de atividade física desde o seu processo histórico, tem refletido as mesmas construções sociais atribuídas a homens e mulheres. Às mulheres, devido ao seu papel reprodutor, consideradas como frágeis ${ }^{11}$, as atividades físicas recomendadas por profissionais de saúde eram restritas àquelas que enaltecessem sua graça, suavidade e beleza, tais como a dança e a ginástica ${ }^{12}$. Já aos homens, que deveriam se opor a tudo que fosse feminino ${ }^{11}$, as atividades consideradas pertinentes eram as de maior impacto, agressivas, e mais rudes e competitivas como as lutas, os jogos de combate e as competições em equipe ${ }^{12}$.

$\mathrm{Na}$ verdade, este modelo, ainda possui um forte impacto nas escolhas de determinadas modalidades esportivas tanto pelos homens quanto pelas mulheres, que ao optarem por atividades que não são indicadas ao seu gênero, enfrentam um grande preconceito por parte da sociedade e da própria família ${ }^{12}$, o que pode influenciar nos modelos de incentivo à prática de exercícios físicos para a promoção da saúde e para a prevenção de doenças pelas instituições governamentais de saúde, principalmente no âmbito nacional.

Diante do exposto, o objetivo deste trabalho foi avaliar a prevalência e as atividades mais praticadas no tempo destinado ao lazer entre os gêneros bem como os principais motivos associa- dos com a prática de atividade física de lazer (AFL) em uma população residente em Campos Elíseos, considerada uma das populações mais pobres do município de Duque de Caxias, área metropolitana do Rio de Janeiro, Brasil.

\section{Material e Métodos}

\section{Amostragem}

Os dados que possibilitaram o desenvolvimento deste trabalho baseiam-se no estudo "Avaliação do estado nutricional, hábitos alimentares e insegurança alimentar no município de Duque de Caxias, Rio de Janeiro: desenvolvimento de um instrumento simplificado para avaliação de consumo alimentar saudável", realizado no período de maio a dezembro de 2005.

Tratou-se de estudo transversal, de base populacional, realizado com a aplicação de entrevistas aos moradores do distrito de Campos Elíseos, pertencente ao município de Duque de Caxias, localizado na região metropolitana do Rio de Janeiro, Brasil. Segundo o ranking do Índice de Desenvolvimento Humano (IDH), o Brasil ocupa a 70 a posição (IDH $=0,80)^{13}$ e o município selecionado foi classificado pelo IDH Municipal (IDH-M) na posição $52^{a}$ (IDH-M=0,75) no ranking dos municípios do estado Rio de Janeiro ${ }^{14}$. Em 2003, Duque de Caxias foi considerado o sétimo município com maior índice de pobreza extrema (14,5\%) no estado do Rio de Janeiro ${ }^{15}$, sendo o distrito Campos Elíseos um dos mais pobres do município ${ }^{16}$.

$\mathrm{O}$ estudo investigou uma amostra probabilística em conglomerados com três estágios de seleção: setor censitário, domicílio e indivíduos. Para determinar o tamanho da amostra, partiuse da prevalência de $14,5 \%$ de pobreza extrema, de forma que uma amostra composta com 1.200 domicílios asseguraria níveis de precisão iguais a $5 \%$. No primeiro estágio, foram determinados, por meio da seleção sistemática, 75 setores censitários dos 322 setores do referido distrito; no segundo estágio, foram selecionados sistematicamente 15 domicílios em cada um dos 75 setores, totalizando amostra com 1.125 domicílios. O terceiro estágio constituiu no sorteio, sendo atribuído número um, para os domicílios que apresentavam pelo menos uma criança ou um adolescente, e dois para domicílios cuja composição familiar era de indivíduos adultos (idade igual ou superior a 19 anos).

Com o objetivo de aumentar a representatividade da amostra e a precisão das estimativas, 
foi realizada antes da seleção dos setores, a classificação destes por renda média mensal do responsável pelo domicílio, o que corresponde a uma estratificação implícita dos setores por renda. Mais detalhes sobre este projeto encontram-se em Salles-Costa et al. ${ }^{17}$.

Foram obtidas informações de 1.085 domicílios, totalizando perdas de 3,6\% referentes a 29 domicílios (recusas, não localização do domicílio ou do chefe da família após três visitas). Da população amostrada, composta por 1275 adultos, $29(2,2 \%)$ foram excluídos devido a respostas inconsistentes ou incompletas, de modo que a amostra final foi composta por 1.246 adultos, sendo $59,5 \%$ mulheres e $40,5 \%$ homens.

A coleta de dados constou de visita domiciliar, com entrevista e exame antropométrico. Todos os entrevistadores passaram por treinamento e os questionários utilizados por um pré-teste e um estudo piloto no local onde se desenvolveu a pesquisa ${ }^{17}$. Os questionários foram digitados por dois bolsistas, e com dupla digitação dos dados.

No momento da entrevista, os participantes foram informados sobre todos os procedimentos realizados (entrevista e avaliação antropométrica), sendo apresentado o termo de consentimento para ser assinado, garantindo o anonimato dos resultados obtidos bem como a recusa da realização dos mesmos. O presente projeto foi aprovado pelo comitê de Ética e Pesquisa da Universidade do Estado do Rio de Janeiro (UERJ).

\section{Variáveis de estudo}

AFL foi definida como qualquer atividade física praticada para melhorar a saúde e/ou a condição física, ou realizada com o objetivo estético ou de lazer, nos três meses anteriores à aplicação do questionário, como recomendado por SallesCosta et al. ${ }^{18}$, sendo avaliada por meio de duas perguntas: na primeira os indivíduos deveriam informar se praticavam ou não $\mathrm{AFL}$, e na segunda, entre os indivíduos praticantes de AFL (resposta afirmativa na pergunta anterior) avaliouse, qual(is) a(s) atividade(s) praticada(s) da relação indicada (bicicleta, musculação, corrida, futebol, hidroginástica, lutas, ginásticas, caminhada, handball, dança, esteira, vôlei, natação, basquete). Para efeitos de análises foram considerados inativos fisicamente os indivíduos que responderam não praticar nenhum tipo de AFL e como ativos fisicamente aqueles que praticavam pelo menos uma AFL.

Para a avaliação das variáveis sócio-demográficas foi aplicado um questionário contendo as seguintes informações: idade (anos); escolaridade (classificada em: analfabetos e ensino fundamental incompleto, ensino fundamental completo, ensino médio e universitário); renda familiar mensal per capita (total de rendimentos da família dividido pelo número de moradores que dependiam desta, expressa em reais e em tercis de renda (para este dado utilizou-se a resposta referida pelo chefe da família ou pelo responsável que permanecia mais tempo em casa e cuidava da alimentação); tempo (em minutos) dispendido em atividade doméstica (lavar roupa, passar, lavar louça e lavar carro); assistência (ou não) de crianças menores de três anos e hábito de fumar (não fumante e fumante).

$\mathrm{O}$ exame antropométrico que incluiu a aferição do peso $(\mathrm{kg})$, da altura $(\mathrm{cm})$ e da circunferência da cintura (CC) em centímetros (duas aferições) de acordo com a padronização de Lohman et al. ${ }^{19}$. O índice de massa corporal (IMC) foi obtido pela divisão do peso pela média das duas aferições da altura, ao quadrado, sendo expresso em $\mathrm{Kg} / \mathrm{m}^{2}$. Os indivíduos foram classificados de acordo com os seguintes pontos de corte: baixo peso (IMC $<18,5 \mathrm{Kg} / \mathrm{m} 2$ ), eutrófico (IMC entre 18,5 e $25 \mathrm{Kg} / \mathrm{m} 2$ ), sobrepeso (IMC entre 25 e $30 \mathrm{Kg} / \mathrm{m} 2$ ) e obeso (IMC $>30 \mathrm{Kg} / \mathrm{m} 2)^{20}$. A CC (média das duas aferições) foi classificada em $\mathrm{CC}<80 \mathrm{~cm}$ para mulheres e $<94 \mathrm{~cm}$ para homens ou CC $\geq 80 \mathrm{~cm}$ para mulheres e $\geq 94 \mathrm{~cm}$ para homens, de acordo com o risco para doenças cardiovasculares, proposto pela $\mathrm{CDC}^{20}$.

\section{Análise de dados}

Estimou-se a as proporções das variáveis entre adultos ativos e inativos fisicamente, de acordo com os gêneros, bem como as atividades físicas realizadas entre os indivíduos ativos, utilizando a razão de prevalência (RP) e seus respectivos intervalos de confiança ao nível de 95\% (IC 95\%), tendo as mulheres como categoria de referência.

Adicionalmente, para avaliar a associação das variáveis sociodemográficas e das medidas antropométricas com a AFL, entre homens e mulheres, foram utilizados modelos de regressão logística para a obtenção dos valores de razões de chance (odds ratio - OR) e seus respectivos IC 95\%, considerando AFL como variável dicotômica (ativos e inativos fisicamente) de desenlace, tendo os indivíduos inativos como categoria de referência. $\mathrm{O}$ modelo final foi ajustado pelas variáveis que apresentaram o nível de significância inferior a $5 \%(\mathrm{p}$-valor $<0,05)$ na análise univariada. 
Todos os procedimentos de análise incorporaram fatores de expansão calibrados. O banco de dados foi elaborado e digitado no programa CSPro 2.5 e a análise de dados realizada no programa Stata $9.0^{21}$.

\section{Resultados}

Nesta população, apenas $1 / 3$ dos adultos praticaram algum tipo de AFL (29,6\%, p-valor $<0,001)$ e os homens se revelaram, significativamente, mais ativos $(43,3 \%)$ do que as mulheres $(20 \%)$ (p-valor $<0,0001)$ de modo que a cada dois homens apenas uma mulher praticou algum tipo de AF no tempo destinado ao lazer $(\mathrm{RP}=1,9$, IC 95\% 1,6-2,2). Observou-se que as atividades mais referidas entre os homens foram o futebol, a corrida, a musculação e a prática de bicicleta ( $\mathrm{p}$-valor $<0,05$ ); entre as mulheres, a caminhada, seguida da prática de bicicleta e da ginástica foram as atividades mais referidas, apesar da ausência de diferença significativa em relação à RP quando comparadas com os homens (Tabela 1).

Quanto à associação entre os fatores sociodemográficos e as medidas antropométricas (Ta- belas 2 e 3) observou-se que, entre os homens, apenas a variável referente ao cuidado de crianças com idade inferior a três anos permaneceu positivamente associada com a maior chance de prática de AFL, enquanto que aqueles com sobrepeso tinham menor chance de praticarem AFL. Entre as mulheres, aquelas que dispendiam menos tempo em atividades domésticas, que não fumavam e que apresentavam maior acúmulo de gordura abdominal (aferida pela CC) tinham, por sua vez, maior chance de serem mais ativas fisicamente no tempo destinado ao lazer.

\section{Discussão}

Poucos são os trabalhos de base populacional com informações sobre atividade física existentes no Brasil. O único inquérito nacional disponível até o presente momento com essas informações consiste na Pesquisa sobre Padrões de Vida (PPV), realizada nas regiões Nordeste e Sudeste ${ }^{7}$. Neste trabalho, constatou-se que apenas $13 \%$ dos indivíduos adultos praticavam pelo menos 30 minutos de atividade física de lazer pelo menos uma vez por semana. No estudo realizado por Gomes et al., em uma amostra represen-

Tabela 1. Razão de Prevalência e seus respectivos intervalos de confiança (IC 95\%) entre modalidades esportivas praticadas no tempo destinado ao lazer de acordo com o gênero (mulheres como categoria de referência). Campos Elíseos/Duque de Caxias - RJ, 2005.

\begin{tabular}{|c|c|c|c|}
\hline Atividade física de lazer & $\begin{array}{c}\text { Homens }(\mathrm{N}=419) \\
\%^{1}(\mathrm{n})\end{array}$ & $\begin{array}{c}\text { Mulheres }(\mathrm{N}=827) \\
\%^{1}(\mathrm{n})\end{array}$ & $\begin{array}{l}\text { Razão Prevalência } \\
\text { (IC 95\%) }\end{array}$ \\
\hline Indivíduos Ativos fisicamente ${ }^{2}$ & $43,3(183)$ & $20,0(186)$ & $1,9(1,6-2,2)$ \\
\hline Modalidades esportivas praticadas & & & $35,0(14,3-85,5)$ \\
\hline Futebol & $21,6(87)$ & $0,6(5)$ & $8,7(2,5-30,5)$ \\
\hline Corrida & $3,3(13)$ & $0,3(3)$ & $4,0(0,7-21,8)$ \\
\hline Vôlei & $0,9(4)$ & $0,2(2)$ & $3,8(1,7-8,1)$ \\
\hline Musculação & $4,7(19)$ & $1,2(10)$ & $3,2(2,2-4,6)$ \\
\hline Bicicleta & $16,9(71)$ & $5,1(43)$ & $2,0(0,1-32,0)$ \\
\hline Basquete & $0,2(1)$ & $0,1(1)$ & $1,0(0,9-11,0)$ \\
\hline Dança & $0,2(1)$ & $0,2(2)$ & $0,9(0,7-1,2)$ \\
\hline Caminhada & $14,4(58)$ & $14,8(120)$ & $0,7(0,1-4,0)$ \\
\hline Hidroginástica & $0,4(2)$ & $0,6(5)$ & $0,5(0,2-1,0)$ \\
\hline Ginástica & $2,1(9)$ & $4,1(34)$ & - \\
\hline Natação & $0,4(2)$ & $0,0(0)$ & - \\
\hline Esteira & $0,0(0)$ & $0,2(2)$ & - \\
\hline Lutas & $0,9(4)$ & $0,0(0)$ & - \\
\hline Handball & $0,0(0)$ & 0,1 (1) & \\
\hline
\end{tabular}

Observação: 1 - Proporções corrigidas pelo desenho da amostra (fator de expansão); 2 - Considerou-se a prática de qualquer atividade física praticada para melhorar a saúde e/ou a condição física, ou realizada com o objetivo estético ou de lazer, nos três meses anteriores à aplicação do questionário. 
Idade $(\text { anos })^{1}$

Escolaridade

Analfabetos e Ensino Fundamental incompleto

Ensino fundamental completo

Ensino médio completo e Universitário

Tercil de renda

$1^{\circ}$ tercil (até 107,14 reais)

$2^{\circ}$ tercil ( $\left.\mathrm{R} \$ 107,15-225,00\right)$

30 tercil (acima de R\$225,01)

Situação conjugal

Casado

Solteiro

Separado/viúvo/desquitado

Atividade doméstica (minutos/dia) ${ }^{2}$

Cuidar de crianças

Não

Sim

Fumo

Sim

Não

IMC $\left(\mathrm{kg} / \mathrm{m}^{2}\right)^{3}$

Baixo-peso

Eutrófico

Sobrepeso

Obesidade

Circunferência da cintura ${ }^{3}$

$<80 \mathrm{~cm}$ (mulheres) e $<94 \mathrm{~cm}$ (homens)

$\geq 80 \mathrm{~cm}$ (mulheres) e $\geq 94 \mathrm{~cm}$ (homens)

Homens Mulheres

\begin{tabular}{|c|c|c|c|}
\hline OR & IC 95\% & OR & IC $95 \%$ \\
\hline 0,97 & $0,96-0,99$ & 1,00 & $0,98-1,01$ \\
\hline
\end{tabular}

$\begin{array}{cccc}1,00 & - & \mathbf{1 , 0 0} & - \\ 1,58 & 0,84-3,00 & 1,65 & 0,84-3,22 \\ 1,12 & 0,56-2,24 & \mathbf{2 , 4 6} & \mathbf{1 , 4 0 - 4 , 3 2}\end{array}$

$\begin{array}{lll}1,00 & - & \mathbf{1}, 00\end{array}$

$0,63 \quad 0,20-2,02 \quad 1,60 \quad 0,75-3,39$

$0,72 \quad 0,25-2,05 \quad \mathbf{2 , 8 6} \quad \mathbf{1 , 3 9 - 5 , 9 2}$

$\begin{array}{llll}1,00 & - & 1,00\end{array}$

$1,64 \quad 0,80-3,36 \quad 1,56 \quad 0,85-2,87$

$1,20 \quad 0,45-3,22 \quad 0,79 \quad 0,41-1,54$

$\begin{array}{llll}0,98 & 0,96-1,00 & \mathbf{0 , 9 9} & \mathbf{0 , 9 8 - 0 , 9 9}\end{array}$

$\begin{array}{lll}\mathbf{1 , 0 0} & - & 1,00\end{array}$

$\mathbf{2 , 6 2} \mathbf{1 , 1 6 - 5 , 9 0} \quad 1,14 \quad 0,60-2,15$

$\begin{array}{lll}1,00 & - & 1,00\end{array}$

$0,62 \quad 0,32-1,21 \quad 3,31 \quad \mathbf{1 , 6 3 - 6 , 7 1}$

$\begin{array}{llll}0,82 & 0,14-4,62 & \mathbf{0 , 1 7} & \mathbf{0 , 0 4}-\mathbf{0}, \mathbf{6 6}\end{array}$

$\begin{array}{llll}1,00 & - & 1,00 & -\end{array}$

$\mathbf{0 , 4 9} \mathbf{0 , 2 7 - 0 , 8} \quad 1,11 \quad 0,64-1,93$

$0,47 \quad 70,19-1,14 \quad 1,13 \quad 0,59-2,13$

$\begin{array}{lll}1,00 & - & \mathbf{1 , 0 0}\end{array}$

$0,52 \quad 0,26-1,04 \quad \mathbf{1 , 7 9} \quad \mathbf{1 , 1 0 - 2 , 9 2}$

Observações: 1 - Variável contínua; 2 - Tempo gasto em atividade doméstica do lar (lavar roupa, passar, lavar louça e lavar carro), avaliada como variável contínua; 3 - Critério de classificação de acordo com $\mathrm{CDC}^{20}$.

tativa do município do Rio de Janeiro, as autoras observaram que $59,8 \%$ dos homens e $77,8 \%$ das mulheres referiram não realizar $\mathrm{AFL}^{9}$.

Apesar da diferença na prevalência de AFL entre homens e mulheres, no Brasil, são poucos trabalhos que integram o conceito de gênero, como destacado por Salles-Costa et al. ${ }^{8}$. Neste estudo, os autores destacaram que além dos resultados revelarem a prevalência elevada de inatividade física entre mulheres, estas referiam maior chance de se engajarem em atividades individuais, que requeriam do corpo menos força física, como a dança, ginástica, caminhada e hidroginástica, enquanto que os homens referiram a prática de atividades físicas coletivas e de caráter competitivo, como futebol, corrida, muscu- lação, lutas, bicicleta e natação. No presente trabalho, se por um lado as escolhas esportivas entre os homens ativos fisicamente, quanto à prática de AFL, reproduziram o modelo de "escolhas masculinas", como pode ser observado na Tabela 1, entre as mulheres não foi possível identificar um padrão considerado feminino, sugerindo que outros fatores interferiram nas escolhas da AFL neste grupo.

Uma hipótese para o padrão observado quanto às escolhas esportivas entre gêneros seria a condição socioeconômica desfavorável da população estudada. Campos Elíseos, local da pesquisa, é um exemplo de uma população marcada por indicadores sociais desfavoráveis, como pode ser verificado no estudo de Salles-Costa et al. ${ }^{17}$ que 
Tabela 3. Razão de chances (OR) e seus respectivos intervalos de confiança (IC95\%) referente à prática de atividade física de lazer entre os gêneros, considerando os indivíduos inativos como categoria de referência (modelo ajustado). Campos Elíseos/Duque de Caxias - RJ, 2005.

\begin{tabular}{|c|c|c|c|c|}
\hline \multirow[b]{2}{*}{ Variáveis Sociodemográficas } & \multicolumn{2}{|c|}{ Homens } & \multicolumn{2}{|c|}{ Mulheres } \\
\hline & OR & IC $95 \%$ & OR & IC $95 \%$ \\
\hline Idade $(\text { anos })^{1}$ & 0,98 & $0,96-1,00$ & - & - \\
\hline \multicolumn{5}{|l|}{ Escolaridade } \\
\hline Analfabetos e Ensino Fundamental incompleto & - & - & 1,00 & - \\
\hline Ensino fundamental completo & & & 1,48 & $0,65-3,37$ \\
\hline Ensino médio completo e Universitário & & & 1,97 & $0,99-3,89$ \\
\hline Tercil de renda & - & - & & \\
\hline $1^{\circ}$ tercil (até 107,14 reais) & & & 1,00 & - \\
\hline $2^{\circ}$ tercil $(\mathrm{R} \$ 107,15-225,00)$ & & & 1,12 & $0,47-2,67$ \\
\hline $3^{\circ}$ tercil (acima de $\mathrm{R} \$ 225,01$ ) & & & 1,49 & $0,59-3,74$ \\
\hline Atividade doméstica (minutos/dia) ${ }^{2}$ & - & - & 0,99 & $0,98-0,99$ \\
\hline Cuidar de crianças & & & - & - \\
\hline Não & 1,00 & - & & \\
\hline Sim & 2,75 & $1,08-6,97$ & & \\
\hline Fumo & - & - & & \\
\hline Sim & & & 1,00 & - \\
\hline Não & & & 2,63 & $1,05-6,57$ \\
\hline \multicolumn{5}{|l|}{$\operatorname{IMC}\left(\mathrm{kg} / \mathrm{m}^{2}\right)^{3}$} \\
\hline Baixo-peso & 0,90 & $0,13-5,91$ & 0,21 & $0,04-1,07$ \\
\hline Eutrófico & 1,00 & - & 1,00 & - \\
\hline Sobrepeso & 0,45 & $0,27-0,78$ & 0,69 & $0,32-1,51$ \\
\hline Obesidade & 0,39 & $0,14-1,07$ & 0,71 & $0,27-1,84$ \\
\hline Circunferência da cintura ${ }^{3}$ & - & - & & \\
\hline$<80 \mathrm{~cm}$ (mulheres) e $<94 \mathrm{~cm}$ (homens) & & & 1,00 & - \\
\hline$\geq 80 \mathrm{~cm}$ (mulheres) e $\geq 94 \mathrm{~cm}$ (homens) & & & 2,72 & $1,15-6,43$ \\
\hline
\end{tabular}

Observações: 1 - Variável contínua; 2 - Tempo gasto em atividade doméstica do lar (lavar roupa, passar, lavar louça e lavar carro), avaliada como variável contínua; 3 - Critério de classificação de acordo com CDC ${ }^{20}$

descreveram que apenas $26,8 \%$ das famílias referiram renda per capita acima de um salário-mínimo, correspondente a 115 dólares na época da coleta de dados (ano de 2005). A segunda hipótese se refere a baixa disponibilidade e a distância das áreas destinadas a prática de exercícios físicos em Campos Elíseos, contribuindo para dificuldades de locomoção, dado a proximidade com áreas de tráfico de drogas e de avenidas de grande movimento de automóveis. Os tipos de áreas para a prática de exercícios seria o terceiro ponto a ser destacado, uma vez que se trata de quadras ou campos de futebol, incentivando a prática de atividades consideradas masculinas em nossa sociedade, tornando as mulheres ainda mais vulneráveis a inatividade física, uma vez que a alternativa seria a prática de exercícios em academias ou clubes (praticamente inexistentes na região) que, junto ao alto valor das passagens, comprometeria a sua fonte de renda.
Aliado a estes fatores, a dupla jornada de trabalho (trabalhar fora e atividades domésticas), como observado no modelo ajustado (Tabela 3), corroborado pelos trabalhos de Eyler et al..$^{22} \mathrm{e}$ Almeida $^{23}$, contribuíram para a inatividade física entre as mulheres uma vez que o tempo disponível para que elas pudessem praticar algum tipo de AFL era mínimo. Por se tratar de uma população de baixa renda, esta situação merece ser ressaltada, pois, se mulheres de maior nível socioeconômico podem delegar seus afazeres domésticos a empregadas, bem como ter acesso a eletrodomésticos que reduzem a carga de trabalho no lar, o contrário, as mulheres de menor poder aquisiti$\mathrm{vo}^{23}$, como as mulheres que residem na população de estudo, tendem a acumular fatores que contribuem para a inatividade física.

Quanto às variáveis antropométricas e sociodemográficas analisadas neste trabalho, as associações encontradas com a inatividade física di- 
feriram entre gêneros. Entre as medidas antropométricas avaliadas, o sobrepeso foi um fator associado à menor prática de exercícios entre os homens, fato também observado no estudo realizado com adultos entre 20 e 59 anos de idade, em Santa Catarina, região Sul do Brasil ${ }^{24}$. Por sua vez, mulheres que não fumavam e com maior CC, a associação com AFL foi positiva, corroborando os resultados observados nos estudos de Pitsavos ${ }^{4}$ e Dias-da-Costa ${ }^{25}$.

A associação entre CC e prática de AFL observada no modelo final merece ser destacada no presente trabalho. Considerando o trabalho de Pinheiro et al. ${ }^{26}$, que utilizou os dados do Programa Nacional de Amostra domiciliar (PNAD) de 1998, os resultados observados revelaram diferença significativa entre os gêneros quanto à procura por serviços de saúde, de modo que as mulheres tenderam a maior busca por serviços preventivos, em oposição aos homens que, por sua vez, procuraram os mesmos por motivo de doença. Desta forma, os resultados encontrados no presente trabalho corroboram para a hipótese de que as mulheres por buscarem mais os serviços de saúde, tendem a receber informações acerca dos riscos do acúmulo de gordura na região abdominal, e com isto, se engajariam mais na prática de alguma AFL, visando reverter este processo.

No presente estudo, não houve associação entre as mulheres ativas ou inativas fisicamente quanto ao cuidado de crianças, apesar desta variável ser descrita na literatura como um fator a mais na rotina da mulher ${ }^{22,23}$, reduzindo o tempo destinado ao seu lazer. Uma possível explicação para este resultado seria a proporção elevada de mulheres com crianças, uma vez que 99,2\% das mulheres avaliadas apresentavam pelo menos uma criança menor de três anos.

Apesar do percentual baixo de perdas do estudo $(3,6 \%)$, uma possível limitação do presente trabalho se refere a menor proporção de homens avaliados, quando comparados com o total de mulheres, decorrente às perdas diferenciais na amostra de adultos selecionados durante a coleta de dados. Para tentar entender este fato, deve ser acrescentado que na primeira visita no domicílio, o entrevistador ordenava a relação dos indivíduos de cada família sendo o adulto considerado como chefe da família, classificado automaticamente com o $1^{\circ}$ número de ordem, seguido do cônjuge ( $2^{\circ}$ número de ordem) e das demais pessoas da família, em ordem decrescente em relação à idade (Exemplo: número de ordem 3 , número de ordem 4 e etc.). Caso o adulto selecionado estivesse ausente após três visitas ao domicílio selecionado, o entrevistador realizava sua substituição pelo segundo adulto indicado pelo número de ordem no registro de controle de substitutos fornecido pelo supervisor de campo. Desta forma, durante os meses de coleta de dados, observou-se que os homens se ausentavam mais dos domicílios, mesmo após as visitas previamente agendadas, levando a substituição destes por outro adulto obedecendo ao número de ordem aleatoriamente gerado com base na etapa e rastreamento dos domicílios, como detalhado por Salles-Costa et al. ${ }^{17}$, e que na sua maioria das vezes era uma mulher.

Uma possibilidade para justificar este fato seria que homens de populações de baixa renda, como os de Campos Elíseos, se ausentam mais dos seus lares devido a sua ocupação laboral, do que as mulheres ${ }^{27}$, ocasionado perdas durante as entrevistas domiciliares. E os homens que permanecem em casa devido ao desemprego ou trabalho informal temporário, tenderiam a auxiliar nas atividades domésticas principalmente saindo de casa para passear com as crianças ${ }^{28}$, contribuindo para a maior chance de praticarem algum tipo de atividade física tais como o futebol e a bicicleta.

Outra limitação se refere ao questionário de atividade física, pois até o momento não existe no Brasil nenhum questionário de atividade física que seja validado. O trabalho de Salles-Costa et al. ${ }^{18}$, no qual este trabalho se baseou para a quantificação da AFL, obteve níveis de confiabilidade variando de discreta a moderada, semelhantes àqueles obtidos em estudos internacionais, como os de Booth et al. ${ }^{29}$ e Philippaerts e Lefevre $^{30}$, sendo então possível afirmar que o questionário utilizado neste estudo apresentou bons níveis de reprodutibilidade.

Concluindo, este trabalho revelou que apenas os homens repetiram o modelo de escolhas de atividades físicas consideradas masculinas, tendo preferência por AFL coletivas e/ou competitivas. As condições sociais desfavoráveis podem ter sido um importante fator que limitou a prática de AFL entre as mulheres, devido à existência de poucos espaços públicos para a prática de exercícios, limitados a quadras e/ou campos de futebol, que incentivam um "modelo masculino" para prática esportiva.

Os resultados aqui apresentados corroboram com a importância do planejamento urbano no incentivo à prática de atividades físicas e esportivas em locais públicos, respeitando as diferenças entre homens e mulheres quanto às suas escolhas e preferências e reforçando a importância de políticas públicas voltadas para a promoção de 
atividades físicas que incorporem o conceito de gênero no planejamento de suas ações, aliadas as desigualdades sociais da população brasileira.

\section{Colaboradores}

SP Sá-Silva participou da concepção, análise de dados e redação do artigo. G Sandre-Pereira participou da concepção e redação do artigo. R Salles-Costa foi responsável pela elaboração do projeto, coordenação do estudo e de todas as etapas da elaboração do artigo.

\section{Agradecimentos}

Esta pesquisa contou com financiamento do Conselho Nacional de Desenvolvimento Científico e Tecnológico (CNPq).

\section{Referências}

1. Trinh OT, Nguyen ND, Dibley MJ, Phongsavan P, Bauman AE. The prevalence and correlates of physical inactivity among adults in Ho Chi Minh City. BMC public health 2008; 8(1):204.

2. Lankenau B, Solari A, Pratt M. International physical activity policy development: a commentary. Public Health Rep 2004; 119(3):352-355.

3. Crespo CJ, Ainsworth BE, Keteyian SJ, Heath GW, Smit E. Prevalence of physical inactivity and its relation to social class in U.S. adults: results from the Third National Health and Nutrition Examination Survey, 1988-1994. Med Sci Sports Exerc 1999; 31(12): 1821-1827.

4. Pitsavos C, Panagiotakos DB, Lentzas Y, Stefanadis C. Epidemiology of leisure-time physical activity in socio-demographic, lifestyle and psychological characteristics of men and women in Greece: the ATTICA Study. BMC public health 2005; 5(1):37.

5. Allender S, Foster C, Boxer A. Occupational and nonoccupational physical activity and the social determinants of physical activity: results from the Health Survey for England. J Phys Act Health 2008; 5(1):104-116.

6. Akamatsu R, Nakamura M, Shirakawa T. Relationships between smoking behavior and readiness to change physical activity patterns in a community in Japan. Am J Health Promot 2005; 19(6):406-409.

7. Monteiro CA, Conde WL, Matsudo SM, Matsudo VR, Bonsenor IM, Lotufo PA. A descriptive epidemiology of leisure-time physical activity in Brazil, 1996-1997. Rev Panam Salud Publica 2003; 14(4):246254.

8. Salles-Costa R, Heilborn ML, Werneck GL, Faerstein E, Lopes CS. Gênero e pratica de atividade física de lazer. Cad Saude Publica 2003; 19(Supl. 2): S325-33.

9. Gomes VB, Siqueira KS, Sichieri R. Atividade física em uma amostra probabilistica da populacao do Municipio do Rio de Janeiro. Cad Saude Publica 2001; 17(4):969-976.

10. Palmer TA, Jaworski CA. Exercise prescription for underprivileged minorities. Curr Sports Med Rep 2004; 3(6):344-348.

11. Scott J. Gênero: uma categoria útil para análise histórica. Educ \& Realidade 1990; 16:5-22. 
12. Adelman M. Mulheres atletas: re-significações da corporalidade feminina. Estudos feministas 2003; 11(2):445-465.

13. Organização das Nações Unidas (ONU). Programa das Nações Unidas para o desenvolvimento. Ranking do IDH. Disponível em: http://www.pnud.org.br/ pobreza_desigualdade/reportagens/index.php?id $01=3120 \&$ lay $=$ pde

14. Organização das Nações Unidas (ONU). Programa das Nações Unidas para o desenvolvimento. Ranking do IDH-M. Disponível em: http://www.pnud.org.br/ atlas/ranking/IDH-2091\%2000\%20Ranking\%20 decrescente $\% 20$ (pelos\%20dados\%20de\%202000)htm.

15. Rocha S, Albuquerque RC. Geografia da pobreza extrema e vulnerabilidade à fome. Seminário especial fome e pobreza. Rio de Janeiro: Estudos e pesquisas; 2003.

16. Instituto Brasileiro de Geografia Estatística (IBGE). Censo demográfico 2000. Disponível em: http:// www.ibge.com.br

17. Salles-Costa R, Pereira RA, Vasconcellos MTL, Veiga GV, Marins VMR, Jardim BC, Gomes FS, Sichieri R. Associação entre fatores socioeconômicos e insegurança alimentar: estudo de base populacional na Região Metropolitana do Rio de Janeiro, Brasil. Rev Nutr 2008; 21(Supl.):99s-109s.

18. Salles-Costa R, Werneck GL, Lopes CS, Faerstein E. Associação entre fatores sócio-demográficos e pratica de atividade física de lazer no Estudo Pró-Saúde. Cad Saude Publica 2003; 19(4):1095-1105.

19. Lohman TG, Roche AF, Martorell R. Antropometric Standardization Reference Manual. Champaing, Illinois: Human Kinetics Books; 1988.

20. Center for Disease Control (CDC). Basics about overweight and obesity. Atlanta: Centers for Disease Control and Prevention; 2002.

21. Stata. Stata Corporation: version 9.2. Texas: college Station; 2007.

22. Eyler AA, Baker E, Cromer L, King AC, Brownson RC, Donatelle RJ. Physical activity and minority women: a qualitative study. Health Educ Behav 1998; 25(5):640-652.
23. Almeida LS. Mãe, cuidadora e trabalhadora: as múltiplas identidades de mães que trabalham. $R e$ vista do Departamento de Psicologia - UFF 2007; 19(2): 411-422.

24. Baretta E, Baretta M, Peres KG. Nível de atividade física e fatores associados em adultos no Município de Joacaba, Santa Catarina, Brasil. Cad Saude Publica 2007; 23(7):1595-1602.

25. Dias-da-Costa JS, Hallal PC, Wells JC, Daltoe T, Fuchs SC, Menezes AM, Olinto MTA. Epidemiology of leisure-time physical activity: a populationbased study in southern Brazil. Cad Saude Publica 2005; 21(1):275-282.

26. Pinheiro RS, Viacava F, Travassos C, Brito AS. Gênero, morbidade, acesso e utilização de serviços de saúde no Brasil. Cien Saude Colet 2002; 7(4):687707.

27. Lavinas L. Empregabilidade no Brasil: Inflexões de gênero e diferenciais femininos. Texto para discussão $n^{\circ}$ 826. Rio de Janeiro: Instituto de pesquisa econômica aplicada, Ministério do Planejamento, Orçamento e Gestão; 2001.

28. Ulysses G. Informalidade no mercado de trabalho brasileiro: uma resenha da literatura. Revista de Economia Politica 2006; 26(4):596-618.

29. Booth ML, Owen N, Bauman AE, Gore CJ. Retest reliability of recall measures of leisure-time physical activity in Australian adults. Int J Epidemiol 1996; 25(1):153-159.

30. Philippaerts RM, Lefevre J. Reliability and validity of three physical activity questionnaires in Flemish males. Am J Epidemiol. 1998; 147(10):982-990 
